\title{
Completeness of data in XRD experiments using Diamond Anvil Cell
}

\author{
D. Tchoń, A. Makal \\ Faculty of Chemistry, University of Warsaw, Pasteura 1, 02-093 Warsaw, Poland \\ dtchon@chem.uw.edu.pl
}

Insufficient coverage of reciprocal space may impede space group determination [1,2], render revealing a crystal structure impossible [3] and conceal or wrongly reveal fine details such as disorder or unusual charge density distribution. [4] Standardised quality checks demand the diffraction pattern to be complete up to a certain resolution, usually $0.6 \AA-1$, for a good reason. [5]

While in majority of X-ray diffraction experiments modern area detectors and multi-axis goniometers allow to quickly scan the reciprocal space, the signal will not be observed if the beam does not have access to sample altogether. [6] This can be caused by a presence of Diamond Anvil Cell (DAC) or any other device which absorbs the beam around the sample. Deficiencies of such kind can be usually fixed by the means of symmetry. [7] The problem stands firm in case of compounds growing in low-symmetry crystal systems. A necessity to collect at least half or quarter of the whole pattern discourages investigators from conducting diffraction experiments, as suggested by the Cambridge Structural Database (CSD) [8] statistics presented in Figure 1.
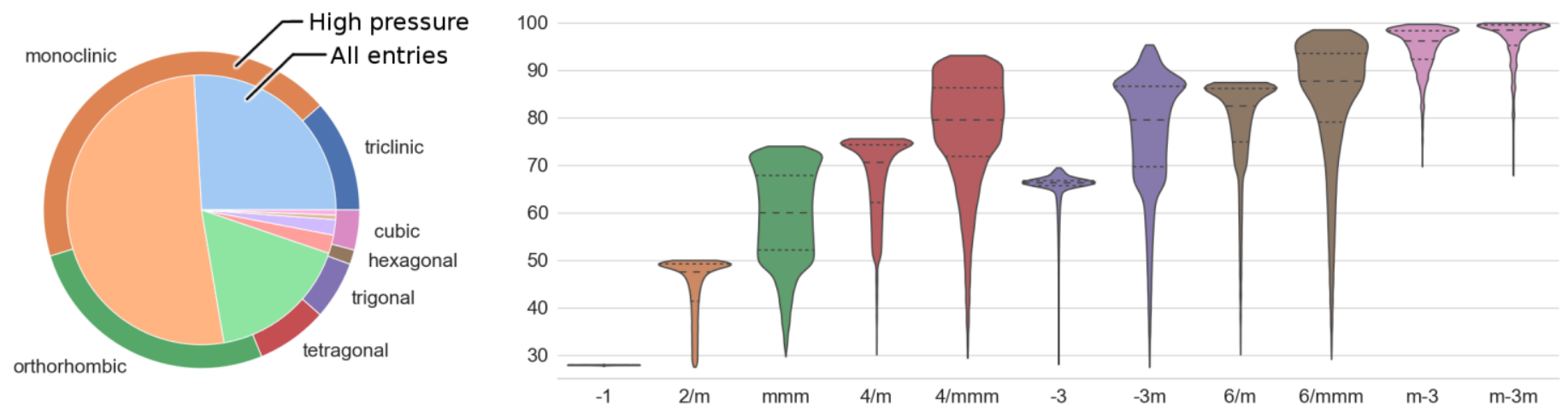

Figure 1. On the left: crystal system frequency for structures deposited in CSD, investigated in ambient (inner circle) and high (outer ring) pressure. On the right: distributions of obtainable diffraction completeness while using MoK $\alpha$ radiation and DAC with a $70^{\circ}$ aperture.

Triclinic and monoclinic crystal systems offer lowest completeness and are clearly under-represented in high-pressure research.

Here we would like to present a comprehensive set of statistics describing a completeness of data in high-pressure experiments. Presented values have been calculated using a series of numerical simulations performed in a custom software. An influence of internal symmetry, crystal orientation and diamond anvil cell geometry on a final data completeness is meticulously analysed. Examples of experimental strategies leading to e.g. complete dataset for monoclinic sample and incomplete dataset for cubic sample are presented. Experimental strategies aiming to increase obtained completeness in various conditions are suggested. While similar estimations have been already suggested, [9] to the best of our knowledge our work is the first comprehensive study of this kind.

[1] Arnold, H., Aroyo, M. I., Bertaut, E. F., Billiet, Y., Buerger, M. J., Burzlaff, H., Donnay, J. D. H., Fischer, W., Fokkema, D. S. et al. (2005). International Tables for Crystallography, Volume A, 5th Edition (reprint), Space-group symmetry, edited by T. Hahn. pp 44-54. Springer.

[2] Sheldrick, G. M. (2015) Acta Cryst A 71, 3.

[3] Yogavel, M., Gill, J., Mishra, P. C. \& Sharma, A. (2007) Acta Cryst D 63, 931.

[4] Takata, M. \& Sakata, M. (1996) Acta Cryst A 52, 287.

[5] Spek, A. L. (2020) Acta Crystallogr. Sect. E 76, 1.

[6] Merrill, L. \& Bassett, W. A. (1974) Review of Scientific Instruments 45, 290.

[7] Binns, J., Kamenev, K. V., McIntyre, G. J., Moggach, S. A. \& Parsons, S. (2016) IUCrJ 3, 168.

[8] Bruno, I. J., Cole, J. C., Edgington, P. R., Kessler, M., Macrae, C. F., McCabe, P., Pearson, J. \& Taylor, R. (2002) Acta Cryst B 58, 389.

[9] Casati, N., Genoni, A., Meyer, B., Krawczuk, A. \& Macchi, P. (2017) Acta Cryst B 73, 584.

These results were submitted to Acta Crystallographica Section A as a research paper.

Keywords: completeness; diamond anvil cell; high pressure; methodology.

Acta Cryst. (2021), A77, C1212 